\title{
Total cross section parameterizations for pion production in nucleon-nucleon collisions
}

\author{
John W. Norbury* \\ NASA Langley Research Center, Hampton, Virginia 23681, USA
}

(Dated: August 8, 2008)

\begin{abstract}
Total cross section parameterizations for neutral and charged pion production in nucleon-nuelcon collisions are compared to an extensive set of experimental data over the projectile momentum range from threshold to $300 \mathrm{GeV}$. Both proton-proton and proton-neutron reactions are considered. Good agreement between parameterizations and experiment is found, and therefore the parameterizations will be useful for applications, such as transport codes.
\end{abstract}

PACS numbers: 13.75. Cs

Many astrophysical gamma rays and neutrinos result from the decay of pions produced in interstellar protonproton (pp) collisions [1]. Most of the muons that travel through the Earth's atmosphere and reach ground level are also the result of pion decay. In modeling astrophysical and cosmic ray sources, one requires an accurate knowledge of pion production cross sections from nucleon-nucleon collisions. Furthermore, because these cross sections are most often used as input to transport codes, it is very useful to have them in a simple, parameterized form, so that the transport codes can run efficiently. Such parameterizations are the subject of this Brief Report. Previous parameterizations have been developed [2] for total pion production cross sections in pp collisions. However, these results were compared to only nine experimental data points, and therefore one did not have much confidence in their validity. In the present work, these same parameterizations are compared to approximately thirty experimental data points. The good agreement obtained gives greater confidence in the use of these parameterizations.

The total inclusive cross section for pion production in proton-proton collisions has been parameterized as [2]

$$
\begin{aligned}
\sigma_{\mathrm{pp} \rightarrow \pi^{+} \mathrm{X}} & =\left(0.00717+0.0652 \frac{\log T_{\mathrm{lab}}}{T_{\mathrm{lab}}}+\frac{0.162}{T_{\mathrm{lab}}^{2}}\right)^{-1} \\
\sigma_{\mathrm{pp} \rightarrow \pi^{-} \mathrm{X}} & =\left(0.00456+\frac{0.0846}{T_{\mathrm{lab}}^{0.5}}+\frac{0.577}{T_{\mathrm{lab}}^{1.5}}\right)^{-1} \\
\sigma_{\mathrm{pp} \rightarrow \pi^{0} \mathrm{X}} & =\left(0.007+0.1 \frac{\log T_{\mathrm{lab}}}{T_{\mathrm{lab}}}+\frac{0.3}{T_{\mathrm{lab}}^{2}}\right)^{-1}
\end{aligned}
$$

where $T_{\text {lab }}$ is the kinetic energy of the projectile and should be specified in $\mathrm{GeV}$ to give $\sigma$ in mb. $X$ represents all other produced particles, appropriate to an inclusive reaction. Below the particle production thresholds, the parameterizations are set to zero. These cross sections are plotted in Figs. $1-3$ as a function of $p_{\text {lab }}$, which is the momentum of the projectile, and compared to experimental data.

\footnotetext{
*Electronic address: john.w.norbury@nasa.gov
}

Consider pp reactions. In reference [2], the parameterizations of equations (1) - (3) were compared only to nine data points from Whitmore [3] at $p_{\text {lab }}=12,19$ and $24 \mathrm{GeV}$. In the present work, they are compared to the Whitmore data at 69,102, 205 and $303 \mathrm{GeV}$, as well as to some other low energy data [4-7], and the data point at $p_{\text {lab }}=954 \mathrm{MeV}$ corresponding to $T_{\text {lab }}=400 \mathrm{MeV}$, of Abd El-Samad et al. [8]. The cross section for the reaction $\mathrm{pp} \rightarrow \mathrm{pp} \pi^{0}$ is $0.1 \mathrm{mb}$. Even though this is an exclusive final state, the exclusive cross section is equal to the inclusive cross section because the energy is below the two pion threshold.

Fig. 1 shows the comparison of equation (1) to the experimental data. The $\mathrm{p}+\mathrm{p} \rightarrow \pi^{+}+\mathrm{X}$ threshold occurs at $T_{\text {lab }}=289 \mathrm{MeV}$, or $p_{\text {lab }}=791 \mathrm{MeV}$. Overall comparison between the parameterization and experiment is good up to a momentum of $300 \mathrm{GeV}$, beyond which the parameterization should not be used, due to lack of data. Fig. 2 shows the comparison of equation (2) to the experimental data. The $\mathrm{p}+\mathrm{p} \rightarrow \pi^{-}+\mathrm{X}$ threshold occurs at $T_{\text {lab }}=600 \mathrm{MeV}$, or $p_{\text {lab }}=1219 \mathrm{MeV}$. This threshold is much larger than for $\pi^{+}$production, which explains why the $\pi^{+}$cross section is larger than the $\pi^{-}$cross section. Again, comparison between the parameterization and experiment is good up to $300 \mathrm{GeV}$. Fig. 3 shows the comparison of equation (3) to the experimental data. The $\mathrm{p}+\mathrm{p} \rightarrow \pi^{+}+\mathrm{X}$ threshold occurs at $T_{\text {lab }}=280 \mathrm{MeV}$, or $p_{\text {lab }}=777 \mathrm{MeV}$. Comparison between the parameterization and experiment is good, although there is some disagreement near $300 \mathrm{GeV}$.

For the above three reactions, the overall agreement between parameterization and experiment is very good. However, the percent differences between parameterization and experiment are biggest in the region very close to threshold, where different physics operates. The worst example is the threshold data point at $\mathrm{p}_{\text {lab }}=1.38 \mathrm{GeV}$ in Fig. 2, where the experimental value is $0.03 \pm 0.01$ mb [5], compared to the parameterization value of 0.97 $\mathrm{mb}$. This difference cannot be seen in the figure due to the very small value of the cross section near threshold. The parameterizations in this paper work very well for momenta up to $300 \mathrm{GeV}$, but they should not be used if accuracy is required very close to threshold.

In the energy region below $6 \mathrm{GeV}$, the neutron-proton (np) cross section is written as a simple multiple of the 


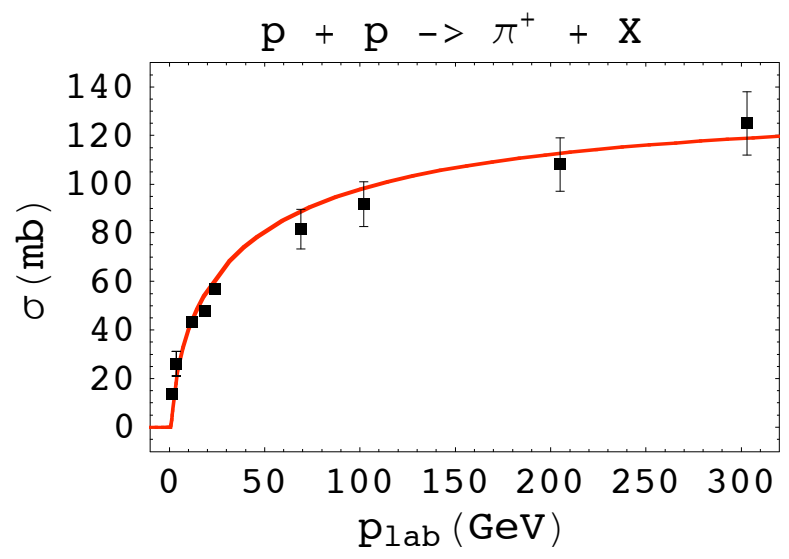

FIG. 1: Total inclusive cross section parameterization of equation (1) versus experimental data [3-5].

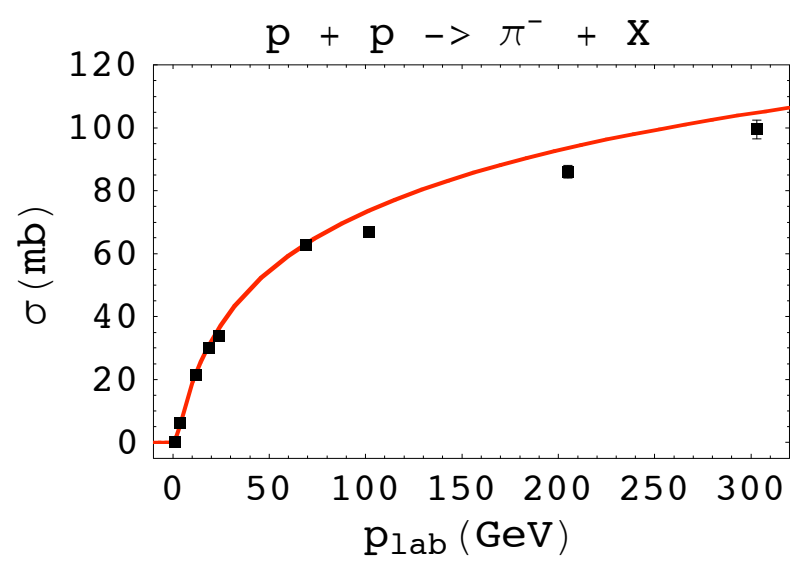

FIG. 2: Total inclusive cross section parameterization of equation (2) versus experimental data [3-5].

pp cross section. The reaction, $\mathrm{n}+\mathrm{p} \rightarrow \pi^{-}+\mathrm{X}$, has been studied by Abdivaliev [7] and the data is shown in Fig. 4, compared to a simple parameterization of the np cross section,

$$
\sigma_{\mathrm{np} \rightarrow \pi^{-} \mathrm{x}}=2.5 \sigma_{\mathrm{pp} \rightarrow \pi^{-} \mathrm{x}}
$$

where $\sigma_{\mathrm{pp} \rightarrow \pi^{-} \mathrm{x}}$ is given in equation (2). The np experimental cross sections for other pion species $\left(\pi^{+}\right.$and $\left.\pi^{0}\right)$ are not available, and therefore parameterizations cannot be developed.

In conclusion, total cross section parameterizations for neutral and charged pion production in nucleon-nuelcon collisions have been compared to an extensive set of experimental data over the projectile momentum range from threshold to $300 \mathrm{GeV}$. Overall, there is good agreement between the parameterizations and experiment in the momentum range up to $300 \mathrm{GeV}$ for proton-proton collisions and up to $6 \mathrm{GeV}$ for neutron-proton collisions.

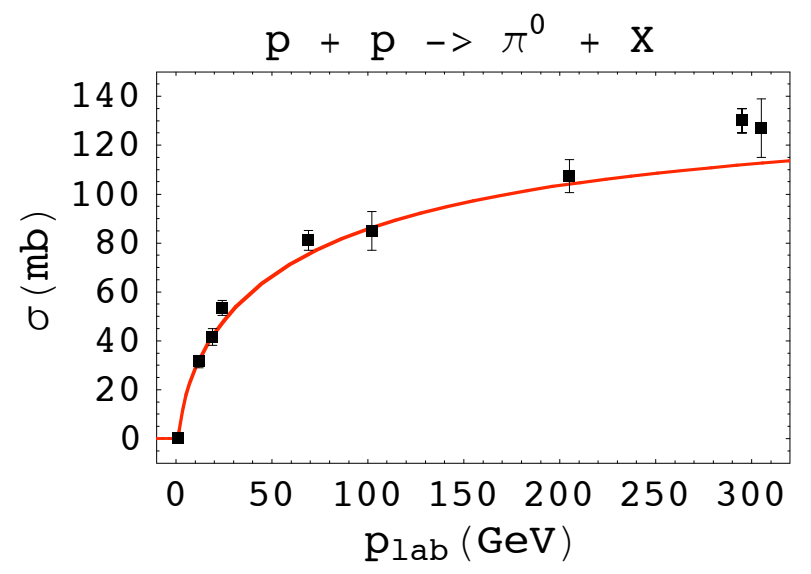

FIG. 3: Total inclusive cross section parameterization of equation (3) versus experimental data [3, 6, 8]. The data points from reference [3] (at $303 \mathrm{GeV}$ ) and reference [6] (at $300 \mathrm{GeV}$ ) have been slightly shifted horizontally so that they do not overlap and can be easily seen.

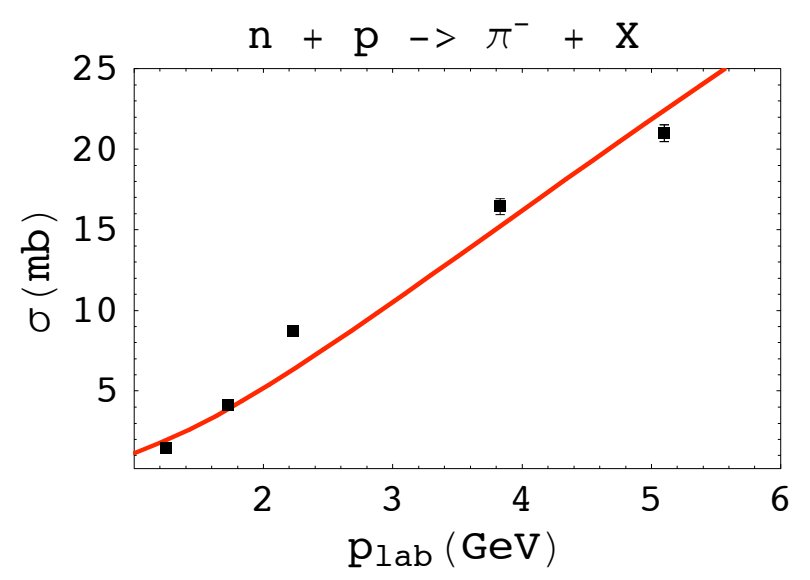

FIG. 4: Total inclusive cross section parameterization of equation (4) versus experimental data [7].

12 for data.

[4] A. C. Melissinos, T. Yamanouchi, G. G. Fazio, S. J. Lindenbaum, and L. C. L. Yuan, Phys. Rev. 1282373 (1962). See Table III for data.
[1] F. Halzen and S. R. Klein, Physics Today 61, 29 (2008).

[2] S. R. Blattnig, S. R. Swaminathan, A. Kruger, M. Ngom, and J. W. Norbury, Phys. Rev. D 62, 094030 (2000).

[3] J. Whitmore, Physics Reports 10, 273 (1974). See Table 
[5] D. R. F. Cochran et al., Phys. Rev. D 6, 3085 (1972). See Table XII for data.

[6] See data listing for the reaction $p+p \rightarrow \pi^{0}+X$, for author named Azimov at http://durpdg.dur.ac.uk/HEPDATA/.

[7] A. Abdivaliev, K. Besliu, S. Gruia, A. P. Ierusalimov,
F. Kotorobai, V. I. Moroz, A. V. Nikitin, D. Pantea, V. N. Pechenov, and Yu. A. Troian, JINR-P1-82-507 (1982). Data available at http://durpdg.dur.ac.uk/HEPDATA/. [8] S. Abd El-Samad et al., Eur. Phys. J. A 30, 443 (2006). 